\title{
Factors Regulating the Encystment Enhancing Activity (EEA) of Acanthamoeba castellanii
}

\author{
By ROBERT A. AKINS, † SUSAN M. GOZS AND THOMAS J. BYERS* \\ Department of Microbiology, The Ohio State University, 484 West 12th Avenue, Columbus, \\ Ohio 43210, USA
}

(Received 18 March 1985)

\begin{abstract}
An extracellular encystment enhancing activity (EEA) greatly stimulates cyst formation by Acanthamoeba castellanii under several different conditions. The activity appears when conditions are suboptimal for growth. EEA does not induce encystment by itself, but enhances differentiation initiated by other factors. EEA titres and their relationship to differentiation are described for encystment induced by: (1) berenil; (2) glucose starvation; and (3) total nutrient starvation. Extracellular EEA was required for maximum encystment by low density cultures in (1) and (2), but not in (3). It was required during the period of visible cyst wall formation rather than for earlier events in encystment. The effectiveness of EEA was reduced under some conditions by spontaneous changes in cellular sensitivity, and by extracellular inhibitors.
\end{abstract}

\section{INTRODUCTION}

Cyst formation by the amoeba Acanthamoeba castellanii is preceded by the extracellular accumulation of an encystment enhancing activity (EEA) when differentiation is induced in a broth medium by berenil or several other inhibitors (Akins \& Byers, 1980). The extent of cyst formation is proportional to the EEA titre even though EEA does not induce encystment by itself. Sufficient EEA titres for maximal cyst formation were produced by high density cultures $\left(\sim 300\right.$ amoebae $\left.\mathrm{mm}^{-2}\right)$, but not by low density cultures $\left(\sim 20-50\right.$ amoebae $\left.\mathrm{mm}^{-2}\right)$. EEA is also necessary for maximal encystment when differentiation is induced in a defined growth medium by glucose starvation (Akins \& Byers, 1980). The EEAs obtained during encystment under the two different conditions share several properties, are interchangeable, and might be identical. Our previous study (Akins \& Byers, 1980) indicated that the biological activity was associated with a low molecular weight compound that was inactivated by snake venom phosphodiesterase. The present study further examines conditions that elevate EEA titres in the medium, reports new evidence for cellular factors that inactivate EEA, investigates the timing of the requirement for EEA under different conditions, and explores changes in sensitivity of the amoebae to EEA.

\section{METHODS}

Organisms, media and culture conditions. Ohio State University clone OS4/7B of Acanthamoeba castellanii (Neff Strain) was used in all experiments. Stocks of amoebae were usually maintained on silica gel (Seilhamer \& Byers, 1978), but some subclones did not survive this treatment. Working cultures were maintained as monolayers (Akins \& Byers, 1980). The broth medium (OGM; Byers et al., 1980) was Neff's yeast extract/proteose-peptone mix. The defined growth medium (DGM-11; Byers et al., 1980) was prepared with the following modifications: amino acids

† Present address: E. A. Doisy Department of Biochemistry, Saint Louis University School of Medicine, St Louis, MO 63104, USA.

Abhreviations: EEA, encystment enhancing activity; DGM-11, defined growth medium: GFDGM, glucosefree DGM-11; NEM, Neff's inorganic encystment medium; OGM, optimal growth medium (broth); ECM, encystment conditioned medium (broth). 
were added as a premixed powder; the final medium included $25 \mathrm{~mm}-\mathrm{KCl} ; \mathrm{KH}_{2} \mathrm{PO}_{4}$ was prepared separately as a $50 \times$ stock and added after autoclaving to avoid precipitate formation during the heating; and glucose was used, but acetate was omitted. Sensitivity to EEA varied spontaneously; therefore, we periodically subcloned the cultures and selected for high encystment under standard bioassay conditions. Encystment conditioned medium (ECM) was obtained from cultures that had encysted to maximal levels in response to berenil in OGM; it has a high EEA titre, but has had the berenil removed by charcoal adsorption (Akins, 1982).

Induction of encystment. Amoebae growing in broth cultures were induced to encyst by addition of $2 \mu \mathrm{g}$ berenil $\mathrm{ml}^{-1}$, by replacement of growth medium with Neff's inorganic encystment medium (NEM; Martin \& Byers, 1976), or by replacement with glucose-free defined growth medium (GFDGM). In some cases, amoebae were transferred from broth to DGM-11 and grown for varying periods before glucose starvation.

Bioassay for EEA. EEA titres were determined using low density test cultures previously shown to give $10-20 \%$ encystment in response to berenil and $65-85 \%$ encystment when high concentrations of EEA were added. In a typical assay of a broth culture, medium was collected, aseptically passed through a $0.22 \mu \mathrm{m}$ pore membrane filter, and then used to replace the growth medium on a test culture grown to 20 amoebae $\mathrm{mm}^{-2}$ in OGM. Tests were done in multiwell tissue culture plates. Medium from a culture induced to encyst with berenil still contained the drug and, therefore, no additional berenil was needed for the assay. Medium from untreated cultures was supplemented with $2 \mu \mathrm{g}$ berenil $\mathrm{ml}^{-1}$. The percentage of cysts determined $4 \mathrm{~d}$ after medium exchange was the EEA titre (Akins \& Byers, 1980).

Ethanol readily precipitates EEA from broth, but not from DGM-11 or NEM. Therefore, samples of DGM or NEM to be tested in the berenil assay were mixed with equal volumes of $2 \times$ OGM and then precipitated with two volumes of cold $95 \%$ ethanol. The precipitate was resuspended in $1 \times$ OGM supplemented with berenil and added to appropriate test cultures. Alternatively, EEA was assayed directly in DGM-11 supplemented with yeast extract and proteose peptone equivalent to $1 \times$ OGM.

Chemicals. Berenil (Ganesag) was generously provided by E. R. Squibb \& Sons, Princeton, NJ, USA, or purchased from Calbiochem-Behring.

\section{RESULTS}

\section{Accumulation of EEA in broth medium}

Berenil added to mid-exponential phase cultures inhibited multiplication, induced accumulation of EEA in the medium, and stimulated high levels of encystment (Fig. $1 a$ ) as observed previously (Akins \& Byers, 1980). EEA also accumulated, although only transiently, as cultures in the absence of berenil ceased to grow exponentially even though cysts did not form (Fig. $1 b$ ).

\section{Temporal order of the berenil and EEA dependent processes}

Berenil did not need to be present continuously for maximum differentiation. Encystment was unaffected by removal of the drug from high density mid-exponential phase broth cultures after a $12 \mathrm{~h}$ exposure. When low density broth cultures were continuously incubated with berenil, maximal encystment of $20 \%$ occurred $40-50 \mathrm{~h}$ later (not illustrated); however, berenil only needed to be present during the first $10-15 \mathrm{~h}$ to achieve this encystment level (Fig. 2). When low density cultures were continuously incubated with berenil plus EEA, maximal encystment was greater and faster, reaching $65-85 \%$ by $20-30 \mathrm{~h}$ (not illustrated). For the maximal response, berenil only needed to be present for the first 5-10 h (Fig. 2). Thus, the requirement for berenil was satisfied well before encystment occurred in each experimental series. Although relatively brief exposures to the drug were sufficient to induce one round of encystment, the cysts formed subsequently hatched in the absence of drug and the emergent amoebae actively replicated.

The EEA requirement differed from the berenil requirement. Low density broth cultures were supplemented with berenil and EEA at time zero and the medium was periodically replaced with fresh medium containing berenil, but lacking EEA. The percentage of cysts was determined at the time of replacement and $4 \mathrm{~h}$ later (Fig. $3 a$ ). Relatively little further encystment occurred after the exchange; maximum encystment of $80 \%$ did not occur unless EEA was present throughout the encystment period. Subsequent experiments indicated that high levels of EEA were not required until cyst wall formation was first visible. This was demonstrated in experiments in which low density cultures initially incubated in broth plus berenil were subsequently supplemented with EEA. A maximum of $20 \%$ encystment was 


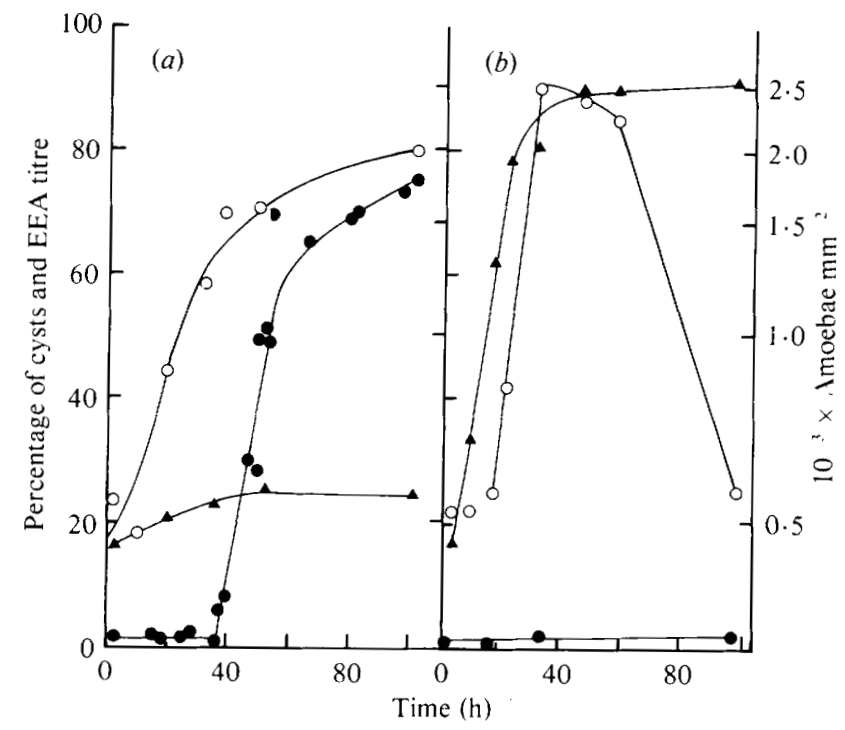

Fig. 1. Variations in EEA titres during growth and encystment in OGM. (a) Mid-exponential phase culture induced to encyst by addition of berenil at $0 \mathrm{~h}$. Data are means of three experiments. (b) Untreated control culture growing at $30^{\circ} \mathrm{C}$. Data are means of two experiments. $\boldsymbol{\Delta}$, Cell density; $\boldsymbol{O}$, percentage of cysts present at the time indicated; $O$, EEA titre, represented as percentage of cysts obtained using a bioassay on the culture medium.

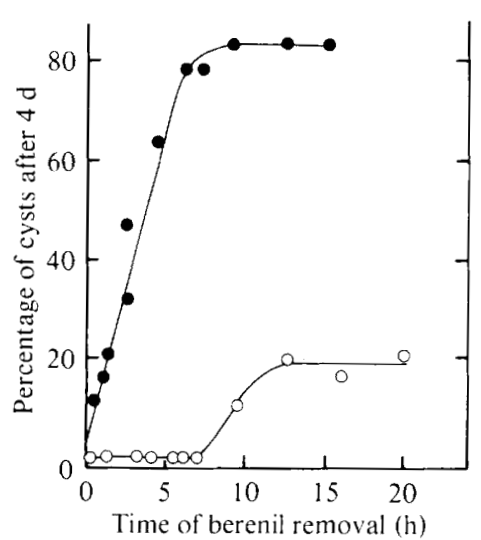

Fig. 2. Effects of medium replacement on berenil induced encystment in low density cultures. Replicate cultures were grown in OGM to 20 amoebae $\mathrm{mm}^{-2}$ and then supplemented with berenil at $0 \mathrm{~h}$. At the times indicated. the medium was replaced with berenil-free OGM (O) or ECM (O). The percentages of cysts are maxima observed before the drug-free cultures excysted and began to multiply. Data for each curve are means of two experiments.

observed in control cultures not receiving EEA, whereas up to $65 \%$ encystment occurred after EEA addition, regardless of the elapsed time at which it was added. However, delays in addition of EEA resulted in delays in encystment; the response time between the addition of EEA and half-maximal encystment decreased from an initial period of $25 \mathrm{~h}$ to a minimum of $10 \mathrm{~h}$ (Fig. $3 b$ ). The minimum response time coincided with the time at which cysts first appeared in low density cultures treated with berenil alone; thus high EEA titres were not required much before the appearance of visible cyst walls. 


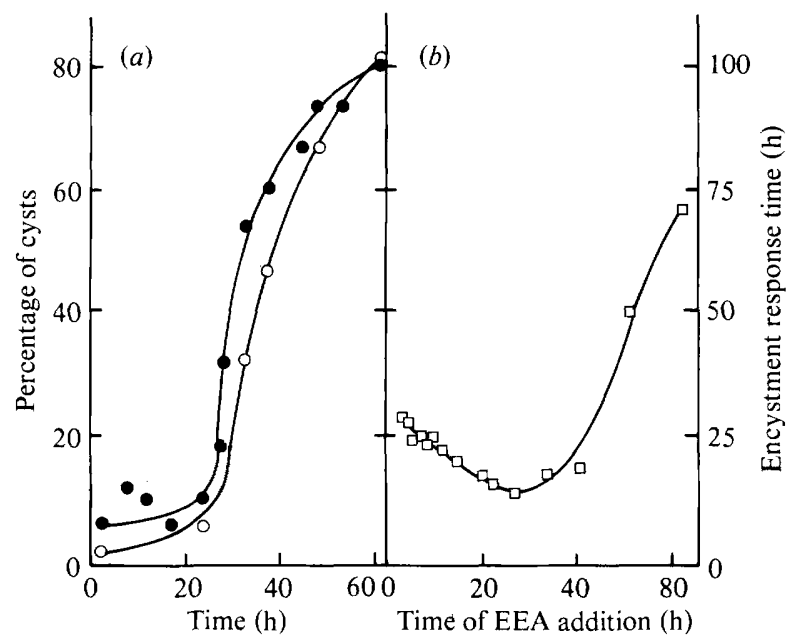

Fig. 3. Effects of EEA removal and addition on encystment in low density cultures. (a) Replicate cultures (20 amoebae $\mathrm{mm}^{-2}$ ) were incubated in berenil-containing $\mathrm{ECM}$. $\mathrm{O}$, Percentage of cysts observed in cultures before medium exchange; all cultures achieved $80 \%$ cysts by $4 \mathrm{~d}$ in the absence of exchange. Percentage of cysts achieved in $4 \mathrm{~d}$ by cultures in which the ECM was replaced at the times indicated with fresh OGM containing berenil, but no EEA. Data are means of two experiments. (b) Low density cultures similar to those in $(a)$ were incubated in fresh OGM plus berenil. At the times indicated, the cultures were shifted to maximal EEA titres by the addition of berenil-containing ECM. The subsequent encystment of each culture was followed for $6 \mathrm{~d}$ and the time for half-maximal encystment determined for each. Encystment response time ( $\square$ ) is the time between ECM (EEA) addition and half-maximal encystment. Data are means of three experiments.

\section{Inactivation of EEA}

EEA produced during late-exponential phase growth was inactivated or removed from the medium during the post-exponential phase (Fig. $1 b$ ). When encystment conditioned medium (ECM ; EEA-containing medium obtained during encystment) was placed on monolayers of late-exponential phase amoebae, the EEA titre initially dropped to low levels, then returned to high levels and eventually dropped to background again (Akins, 1982). Similar experiments demonstrated that the decreases in EEA titre were due to substances released from the amoebae into the medium. ECM was placed on monolayer cultures for $2 \mathrm{~min}$ or less, and then recovered and filtered to remove amoebae. Replicate samples were incubated at $30^{\circ} \mathrm{C}$ for various intervals, frozen, and later assayed for EEA. The titre decreased during the cell-free incubation to a minimum after $7 \mathrm{~h}$ and then returned to its initial level upon further incubation (Fig. 4). The ability of factors produced by different clones of amoebae to transiently inactivate EEA in this assay was inversely correlated with the concentrations of EEA required to produce enhanced encystment in low density cultures (Akins, 1982). The decrease in EEA titre was prevented if a proteolytic enzyme was present during the period of cell-free incubation; rather, an increase in titre occurred (Fig. 4). A similar increase was observed if ECM that had not been exposed to late-exponential phase monolayers was treated with proteolytic enzymes (Fig. 4). Thus, the inactivating factor is protein produced by late-exponential phase amoebae and still present during the post-exponential phase. Preliminary cell fractionation studies suggest that EEAinactivating proteins are found in a subcellular fraction containing membranes and mitochondria (Akins, 1982). Since EEA titres depressed by interaction with proteins from exponential phase amoebae spontaneously return to higher levels (Fig. 4), the inactive complex must dissociate or the protein must be destroyed.

\section{EEA titres and encystment during glucose starvation in defined growth medium}

High density mid-exponential phase cultures growing in defined growth medium containing glucose (DGM-11) encysted when the medium was replaced with glucose-free DGM-11 


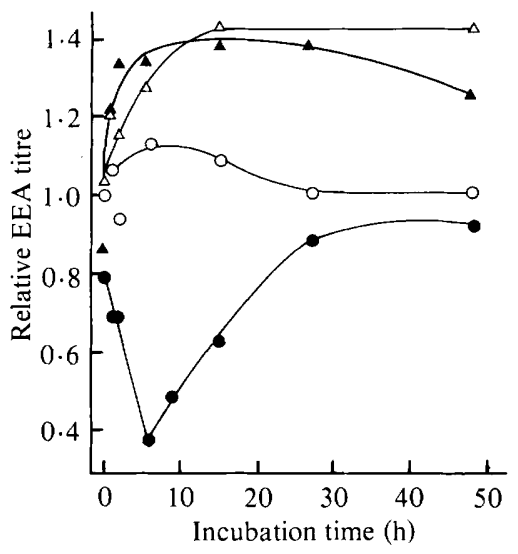

Fig. 4. Interaction of EEA with factors released from exponential phase amoebae. Cultures were grown in OGM to 900 amoebae $\mathrm{mm}^{-2}$ and the medium was then replaced with berenil-free ECM. Within 2 min the ECM was recovered, aseptically filtered, distributed to sterile vials, and incubated at $30^{\circ} \mathrm{C}$ for the indicated times. Half of the vials $(\boldsymbol{A})$ were supplemented with $100 \mu \mathrm{g}$ pronase $\mathrm{ml}^{-1}$ during the incubation; the remainder of the vials had no enzyme (O). Control ECM that had not been exposed to the exponential phase amoebae was also incubated with $(\triangle)$ and without $(O)$ pronase. EEA titres remaining after incubations were determined by bioassay and expressed relative to titres at zero time (relative EEA titre). Data for protease-treated media are means of two experiments; data for untreated media are means of five to fifteen experiments.

(GFDGM). Multiplication was limited to one to two additional doublings (Fig. 5a) and synchronous encystment occurred after $45-60 \mathrm{~h}$ (Fig. $5 b$ ). A relatively high EEA titre was associated with exponential phase growth in DGM-11. Replacement of medium from growing cultures with fresh GFDGM reduced EEA levels to background, but the titres increased before maximal encystment (Fig. $5 b$ ). The maximum percentage of cysts achieved during glucose starvation before cell lysis was dependent on culture density (Fig. 5c) and EEA titre (not shown). The amoebae used in the present study were more fragile than those used previously when the rate of encystment, rather than the maximum percentage of cysts, was dependent on culture density (Akins \& Byers, 1982).

\section{Changes in sensitivity to EEA during prolonged culture in DGM-11}

Low density cultures recently subcultured in DGM-11 from broth and then starved of glucose, or transferred directly from broth to GFDGM, encysted well when supplemented with EEA (Fig. 6a). Low density cultures adapted to growth in DGM-11 did not respond to EEA in GFDGM, nor to berenil plus EEA in broth (Fig. $6 a, b$ ). The sensitivity lost by DGM-adapted cultures was restored by subculture in broth (Fig. 6a). Amoebae adapted to DGM-11 still encysted in GFDGM at high culture densities and encystment was dependent on EEA. If the medium of high density cultures starving in GFDGM was replaced just before the time at which cysts normally began to appear, encystment was delayed $40-50 \mathrm{~h}$ (Fig. 7 a). Earlier replacements were not effective. The delay could be reduced or eliminated by replacing the original medium, or by substituting medium containing EEA (Fig. $7 a$ ). Diluted high density cultures also showed a delay that could be corrected by addition of EEA (Fig. 7b). Thus diluted amoebae responded to EEA even though low density cultures did not (Fig. $6 a, b$ ).

\section{EEA and encystment in inorganic encystment medium}

Induction of encystment by starvation in NEM is the most effective way of obtaining cysts. It typically works even with clones that do not respond to berenil or glucose starvation (Akins, 1982). Encystment in NEM was culture density dependent (Fig. 8a); however, there was little evidence for EEA in the medium during encystment at high densities (Fig. 8b), and cultures diluted to lower densities encysted well without the addition of EEA (Fig. 8a). When samples of 


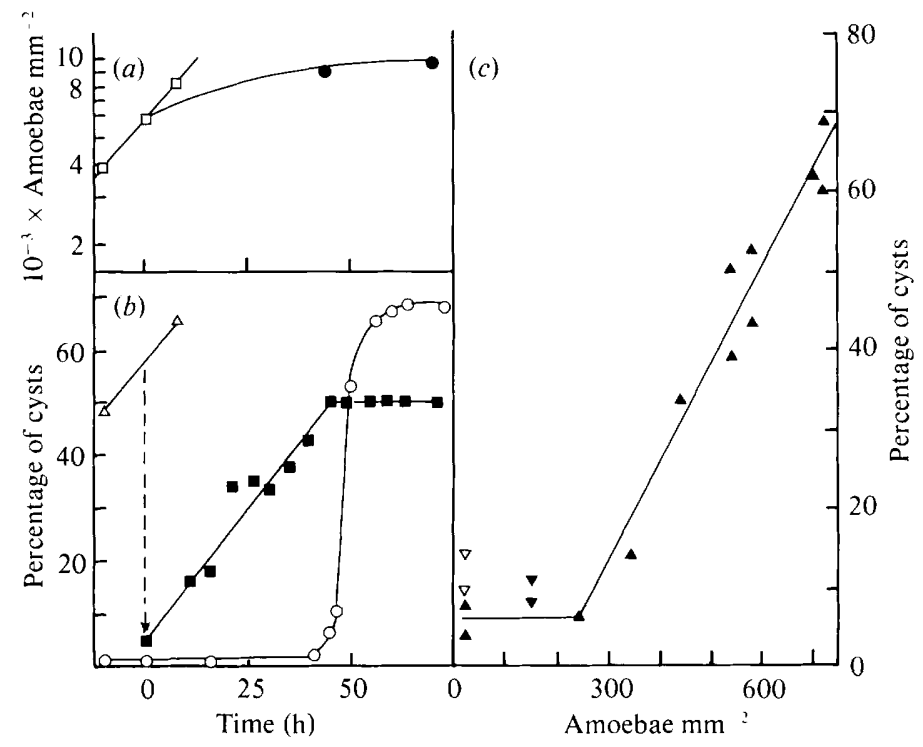

Fig. 5. Encystment and EEA titres during glucose starvation in GFDGM. DGM-adapted cultures were grown in DGM to 600 amoebae $\mathrm{mm}^{-2}$ and the medium was replaced at $0 \mathrm{~h}$ with fresh GFDGM. (a) Increase in culture density in DGM $(\square)$ and after replacement with GFDGM $(O)$. (b) Percentage of cysts in the culture $(O)$ and EEA titre in the medium before $(\triangle)$ and after $(\square)$ the medium replacement. The dashed line indicates the decrease in EEA titre caused by medium replacement. (c) Maximum percentage of cysts obtained $60 \mathrm{~h}$ after initiation of glucose starvation. $\boldsymbol{\Delta}$, Various initial culture densities; $\nabla$, cultures grown to 660 cells $\mathrm{mm}^{-2}$ diluted to 20 cells $\mathrm{mm}^{-2}$ at the initiation of stavation; $\nabla$, cultures diluted from 700 to 150 cells $\mathrm{mm}^{-2}$ after $35 \mathrm{~h}$ of starvation. Experiments were repeated three or more times; data are averages of three replica samples from representative experiments.

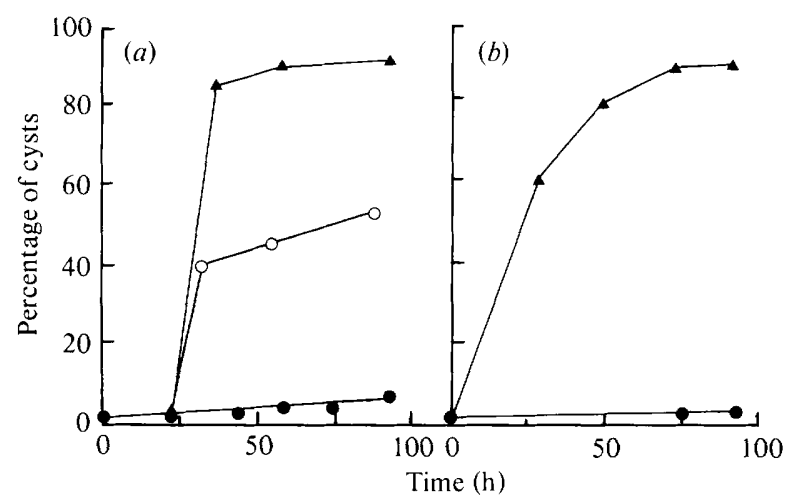

Fig. 6. Loss of EEA sensitivity in low density cultures following prolonged growth in DGM. (a) Encystment during glucose starvation in GFDGM containing EEA. (b) Encystment in OGM containing EEA plus berenil. - DGM-adapted cultures; $\boldsymbol{\Delta}$, cultures maintained in OGM and then grown for one subculture in DGM before starvation; $O$, DGM-adapted cultures that had been returned to OGM for 2 weeks before glucose starvation in GFDGM. In all cases, starvation was initiated at 20 amoebae $\mathrm{mm}^{-2}$. Experiments were repeated twice; data are mean values for replicates within one experiment.

medium taken at various times during encystment at high densities were added to encystment conditioned broth, the broth EEA titre decreased. The EEA-inactivating activity thus increased during the encystment period (Fig. $8 b$ ). 


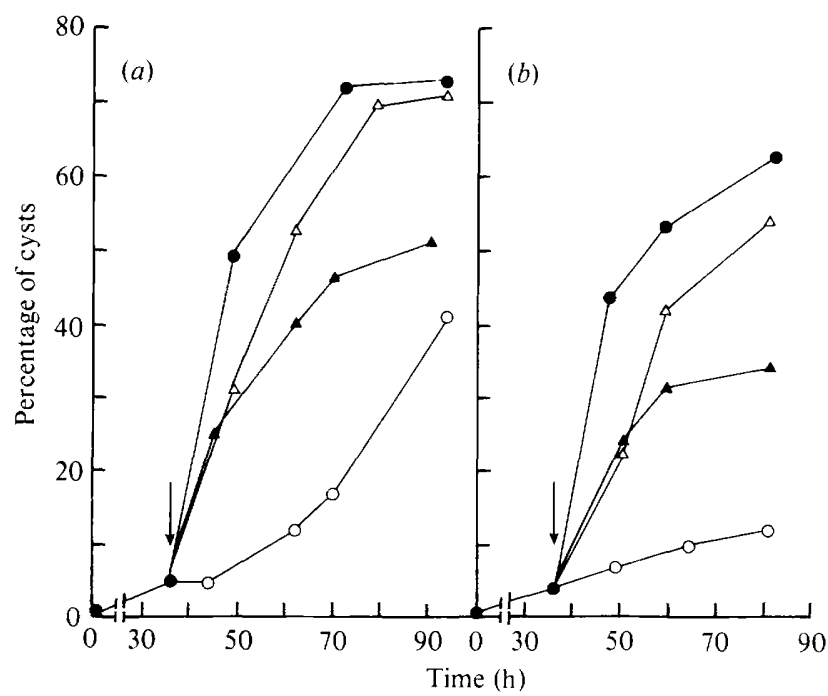

Fig. 7. Effects of medium replacement on glucose starvation-induced encystment in high density cultures. (a) DGM-adapted cultures were grown to 700 amoebae $\mathrm{mm}^{-2}$ and the medium was then replaced with GFDGM. (b) Cultures were as in $(a)$, but were diluted from 700 to 100 amoebae $\mathrm{mm}^{-2}$ at the time of medium exchange. The control culture was diluted, but without medium exchange. After $36 \mathrm{~h}$ of glucose starvation, the medium was replaced (arrow) with the same medium after aseptic filtration $(\triangle)$, with fresh GFDGM $(O)$, or with filtered medium obtained from a culture that had encysted actively in GFDGM and contained a high EEA titre (A). Undisturbed control cultures. The experiment was repeated three times; data are individual values from a representative experiment.

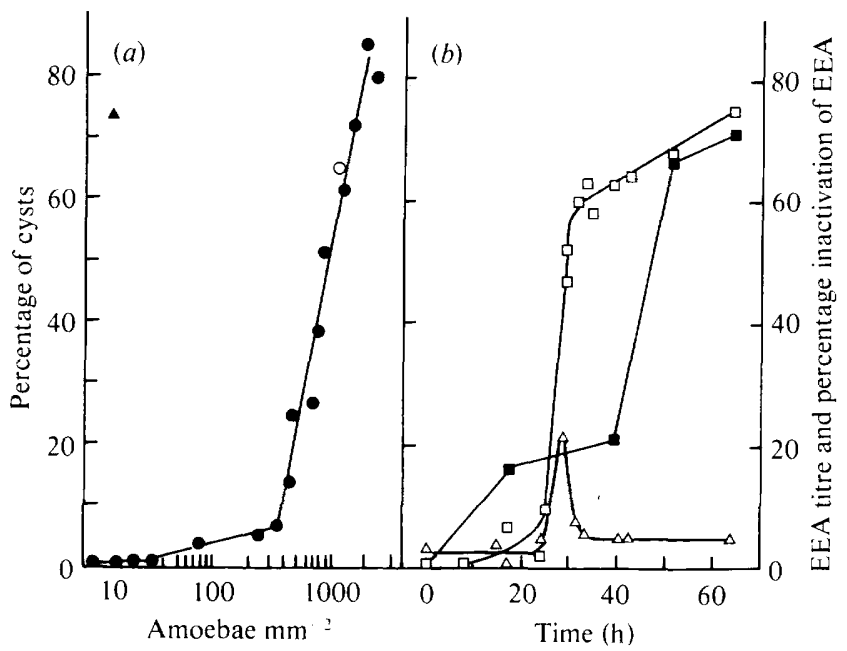

Fig. 8. Encystment and EEA titres during total nutrient-starvation in NEM. Cultures were grown in OGM to the indicated initial culture densities and the medium was then replaced with NEM. $(a)$ Maximum percentage of cysts obtained $4 \mathrm{~d}$ after initiation of starvation. Various initial culture densities: $\boldsymbol{\Delta}$, culture grown to 1400 amoebae $\mathrm{mm}^{-2}$ and diluted to $14 \mathrm{~mm}^{-2}$ at the initiation of starvation; $O$, culture grown to 20 amoebae $\mathrm{mm}^{-2}$ and concentrated to $1100 \mathrm{~mm}^{-2}$ at the beginning of starvation. (b) $\square$. Percentage of cysts during starvation of a culture at 1200 amoebae $\mathrm{mm}^{-2}$. Data are means of replicate samples in one experiment $\triangle$. EEA titre in the medium; $\square$, relative EEAinactivating activity of the medium as determined by assaying the decrease in total EEA titre that occurred when samples of the NEM medium were incubated with equal volumes of EEA-containing OGM for $8 \mathrm{~h}$ at $37^{\circ} \mathrm{C}$. Data are means of two experiments. 
Low density broth cultures produced only $11 \%$ cysts during starvation in NEM, but the response increased to $>60 \%$ if the amoebae were incubated for $2 \mathrm{~h}$ in DGM-11 (or GFDGM) at $\mathrm{pH} 8.5$ before NEM starvation. The high $\mathrm{pH}$ treatment did not enable cultures to encyst in berenil supplemented broth, where the continuous presence of EEA was required (Fig. $3 a$ ). Low density broth cultures were also able to encyst effectively in NEM without the addition of EEA if they were concentrated to 1100 amoebae $\mathrm{mm}^{-2}$ (Fig. 8a).

\section{DISCUSSION}

The appearance of EEA is associated with inhibition of multiplication by antibiotics or glucose starvation, and with the late-exponential phase of growth. EEA does not inhibit cell multiplication nor stimulate differentiation by itself (Akins \& Byers, 1980). For berenil-induced encystment, the initiation of differentiation precedes the period during which EEA is most effective (Fig. 2; Fig. 3b). It is unknown whether berenil simply inhibits multiplication or has direct effects on other processes, such as stimulation of EEA production, that are important for encystment. EEA is closely associated with cyst wall formation in both berenil induced (Fig. $3 a$, b) and glucose starvation induced encystment (Fig. 7a,b). The in vivo function of EEA remains an enigma, however, especially in the absence of evidence for a role during encystment in NEM. One possibility is that EEA is an essential developmental morphogen or intermediate in cyst wall formation, but that significant extracellular titres occur only during encystment in media such as GFDGM and broth which contain a variety of nutrients. We have extensive evidence that subclones from unselected populations exhibit extreme variation in sensitivity to berenil and other drugs. Production of extracellular EEA by amoebae more sensitive to a particular detrimental factor or condition might promote population survival by enhancing differentiation in less sensitive amoebae. In contrast, total starvation in NEM is a very strong stimulus that affects subclones more uniformly. More importantly, by eliminating the external supply of nutrients, it probably forces amoebae to internally sequester essential metabolites, possibly including EEA.

The stimulation of encystment in low density cultures when DGM-11, which has a high $\mathrm{pH}$, is added before incubation of the amoebae in NEM is not simply due to a change in pH. NEM has the same high $\mathrm{pH}$, but does not induce the encystment by itself. The stimulation could be related to ion transport, since certain divalent cations are known to favour encystment (Neff et al., 1964; Band, 1963; Chagla \& Griffiths, 1974). We were unable to activate encystment in low density cultures adapted to growth in DGM-11. Obviously, the factors in DGM-11 that activate encystment by low density cultures in NEM are not sufficient. Apparently the adapted amoebae have developed an encystment deficiency. The deficiency can be corrected by subculturing in broth, but can also be overcome by concentrating amoebae to higher densities (see below).

Low density cultures that encyst poorly in NEM differentiate very well if simply concentrated in the medium. High density cultures retain their ability to encyst upon dilution in NEM (Fig. $8 a$ ). Thus, a stable cellular transformation in the ability to respond to NEM seems to occur at high densities. A similar transformation was found for DGM-11 adapted cultures which did not encyst after growth to low densities, even if supplemented with EEA (Fig. $6 a, b$ ), but did encyst at higher densities. The latter cultures then retained the ability to encyst in response to EEA and berenil when they were diluted to low densities (Fig. $7 c$ ). Although the activation of encystment that occurred at high culture densities in NEM and GFDGM was retained upon dilution, it was independent of EEA in the inorganic medium (NEM) and dependent on EEA in the nutrientcontaining medium (GFDGM).

Factors that interact with EEA and lower the effective titre are present in broth medium during the late-exponential growth phase (Fig. $1 b$ ), during berenil induced encystment (Fig. 4), and during NEM induced differentiation (Fig. 8). One active component of EEA has a molecular weight of 500 or less and can be inactivated by bacterial alkaline phosphatase, $5^{\prime}$ nucleotidase, $3^{\prime}$-nucleotidase and snake venom phosphodiesterase. Chromatography of EEA on DEAE-Sephadex also reveals bioactivity associated with high molecular weight forms (Akins, 1982). Presumably the latter include the complexes with inactivating proteins. 
Further progress clearly requires the identification of EEA. S. M. Gozs (unpublished results) has found that simple sugar phosphates, including glucose 1-phosphate, galactose 1-phosphate and fructose 1-phosphate, can substitute for EEA in GFDGM. In contrast, glucose, galactose and fructose inhibit differentiation when added to this encystment medium. The sugar phosphates are not effective, however, in the broth-berenil induction system and as yet we have no evidence that they are components of the natural EEA produced in GFDGM.

This work was supported by National Science Foundation grant PCM 8011696 to T.J.B.

\section{REFERENCES}

Akins, R. A. (1982). Differentiation of Acanthamoeba castellanii: evidence for a developmental morphogen and exploratory genetic approaches. $\mathrm{PhD}$ dissertation, The Ohio State University. Dissertation Abstracts International 42, 3951 B.

AKINS, R. A. \& BYERS, T. J. (1980). Differentiation promoting factors induced in Acanthamoeba by inhibitors of mitochondrial macromolecule synthesis. Developmental Biology 79, 126-140.

BAND, R. N. (1963). Extrinsic requirements for encystation by the soil amoeba, Hartmannella rhysodes. Journal of Protozoology, 10, 101-107.

Byers, T. J., Rudick, V. L. \& Rudick, M. J. (1969). Cell size, macromolecule composition, nuclear number, oxygen consumption and cyst formation during two growth phases in unagitated cultures of Acanthamoeha castellanii. Journal of Protozoology 16, 693699.

Byers, T. J., AKINS, R. A., MAYNARD, B. J., LEFKEN, R. A. \& MARTIN, S. M. (1980). Rapid growth of
Acanthamoeba in defined media; induction of encystment by glucose-acetate starvation. Journal of Protozoology 27, 216-219.

Chagla, A. H. \& Griffiths, A. J. (1974). Growth and encystation of Acanthamoeba castellanii. Journal of General Microbiology 85, 139-145.

MARTIN, S. M. \& ByERS, T. J. (1976). Acid hydrolase activity during growth and encystment in Acanthamoeba castellanii. Journal of Protozoology 23, 608613.

NeFF, R. J., Ray, S. A., Benton, W. F. \& Wilborn, M. (1964). Induction of synchronous encystment (differentiation) in Acanthamoeba sp. Methods in Cell Physiology 1, 55-83.

Seilhamer, J. J. \& Byers, T. J. (1978). Mutants of Acanthamoeba castellanii resistant to erythromycin, chloramphenicol, and oligomycin. Journal of Protozoology 25, 486-489. 